\title{
Emerging role of RUNX3 in the regulation of tumor microenvironment
}

\author{
Sarala Manandhar \& You Mie Lee* \\ Laboratory of Vascular Homeostasis Regulation, BK21 Plus KNU Multi-Omics based Creative Drug Research Team, Research Institute of \\ Pharmaceutical Sciences, College of Pharmacy, Kyungpook National University, Daegu 41566, Korea
}

\begin{abstract}
A number of genes have been therapeutically targeted to relieve cancer, but cancer relapse is still a growing issue. The concept that the surrounding tumor environment is critical for the progression of cancer may foster an answer to the issue of cancer malignancy. Runt domain transcription factors (RUNX1, 2, and 3) are evolutionarily conserved and have been intensively studied for their roles in normal development and pathological conditions. During tumor growth, a hypoxic microenvironment and infiltration of the tumor by immune cells are common phenomena. In this review, we briefly introduce the consequences of hypoxia and immune cell infiltration into the tumor microenvironment with a focus on RUNX3 as a critical regulator. Furthermore, based on our current knowledge of the functional role of RUNX3 in hypoxia and immune cell maintenance, a probable therapeutic intervention is suggested for the effective management of tumor growth and malignancy. [BMB Reports 2018; 51(4): 174-181]
\end{abstract}

\section{INTRODUCTION}

A large number of oncogenes and tumor suppressor genes were identified and therapeutically targeted for effective care of cancer patients in past two decades, which have also extended survival of cancer patients. But the relapse of cancer due to increased resistance to therapeutic intervention has raised another critical question. With this scenario, the new concept is emerging that appreciates the fact that cancer maintenance and expansion is critically regulated by signals from the microenvironment $(1,2)$. Tumors are complex tissues i.e, structures comprising not only malignant cells, but also contain genetically stable stromal cells. Cells making up the

${ }^{*}$ Corresponding author. Tel: +82-53-950-8566; Fax: +82-53-9508557; E-mail: lym@knu.ac.kr

https://doi.org/10.5483/BMBRep.2018.51.4.033

Received 20 January 2018

Keywords: HIF-1 $\alpha$, Hypoxia, Immune cell, RUNX3, Tumor microenvironment structural framework of tumors include specialized cells of the connective-tissue family called fibroblasts, endothelial cells and immune cells. Together with products such as the extracellular matrix (ECM), these cellular interactions drive the initiation, growth, and metastasis of tumors (1). Tumor hypoxia is another critical microenvironmental state that results because of an inadequate oxygen supply in growing solid tumors. Hypoxia compromises biological functions and is associated with malignancy and cancers that are refractory or resistant to treatment (3).

The Runt-related transcription factor (RUNX) family is evolutionarily conserved from simple to complex organisms, suggesting its significant role in developmental and biological processes. It is well described as developmental regulators and its involvement in human neoplasia is also been documented. The Runt domain transcription factors are composed of a larger DNA-binding subunit, $\alpha$, and a smaller non-DNA-binding subunit, $\beta$ (known as core binding factor $\beta, C B F \beta$ (4). RUNX1, RUNX2, and RUNX3, the three members of the Runx family genes, encode $\alpha$ subunits. RUNX1 is important for the generation of hematopoietic stem cells, and it is also illustrated to be involved in leukemia $(5,6)$. RUNX2 is essential for bone development and has oncogenic potential $(7,8)$. RUNX3 is associated with multiple developmental functions and differentiation of immune cells such as CD8-lineage T cells (5, 9), TrkC-dependent dorsal root ganglion neurons (10), and it also functions as a major tumor suppressor (11-16). In addition to its role as a tumor suppressor, recent researchers have depicted it as a tumor promoter in various cancers (17-19).

In this review, we discuss our overall knowledge on the emerging role of RUNX3 in the regulation of the tumor microenvironment. The instructions harnessed in the regulatory mechanism and modulation can be instrumental for the development of more effective anticancer therapies.

\section{RUNX3 IN TUMORIGENESIS}

RUNX3 is expressed in a wider range of tissues with multiple roles. RUNX3 is a well-recognized tumor suppressor of gastric, colon and many other forms of solid tumors. The RUNX3-deficient phenotype is described as a cause for hyperplasia of the gastric mucosa (16), while severe limb 
ataxia is also illustrated with a Runx3 deletion (20). The tumor suppressive role for RUNX3 is further strengthened by an inactivated RUNX3 on hemizygous deletion, promoter hypermethylation, histone modification and frequent protein mislocalization (14, 21-24). Recently, RUNX3 is suggested to inhibit migration and invasion of melanoma cells through enhancing the formation of stress fibers and mature focal adhesion and ECM protein production regulating the cell shapes (25).

Recently, in addition to established tumor suppressive role of RUNX3, its oncogenic role is also being revealed in certain cancer subsets. All three RUNX genes were first shown to be associated with MYC proto-oncogene (MYC) thus promoting leukemogenesis (26). Later its oncogenic behavior was verified in basal cell carcinoma with overexpressed RUNX3 in cancers compared to the normal epidermis (27). Likewise, RUNX3 overexpression is observed to increase proliferation and tumorigenesis in ovarian cancer, head, and neck squamous cell carcinoma and Ewing sarcoma (18, 28, 29). All these observations suggest the cell type and contextual-dependent behavior of RUNX3 as a tumor suppressor or a promoter.

\section{TUMOR MICROENVIRONMENT}

Conventional cancer therapies are more focused on eliminating proliferating cancer cells, while recent advances in cancer biology have emphasized the critical importance of the surrounding environment in initiation, progression, and metastasis of cancer $(30,31)$. Development of a tumor involves encompassing the proliferating tumor cells, the tumor stroma, blood vessels, infiltrating immune cells and various types of macromolecules comprising the extracellular matrix (ECM) and a large number of secreted cytokines and molecules. The tumor microenvironment (TME) develops during the progression of the tumor as a unique environment, through interaction of tumor cells with the surrounding host cells and their secretions. It plays not only pivotal role in tumor initiation, but also facilitates progress of cancer i.e., metastasis $(32,33)$. The microenvironment of the tumor is often hypoxic. As the mass of the tumor increases, the inner side of the tumor grows far beyond the existing blood supply. Angiogenesis may reduce this effect, but at more than $50 \%$ of locally advanced solid tumors, the oxygen partial pressure is less than $5 \mathrm{mmHg}$ (oxygen partial pressure of the venous blood $(40 \mathrm{mmHg}$ ) (34, 35). The hypoxic microenvironment has been shown to strongly influence the dialogue between tumor cells and nonmalignant stromal cells, thus inducing changes in the proteome of these cells and facilitating tumor propagation by enabling the cells to adapt in a nutrient-deficient hostile microenvironment (36) (Fig. 1).

Tumor-associated macrophages (TAMs), abundant in most of the human and mouse tumor microenvironments, have primarily pro-tumorigenic abilities (37). They are reported as potent partners for cancer cell migration, invasion, and

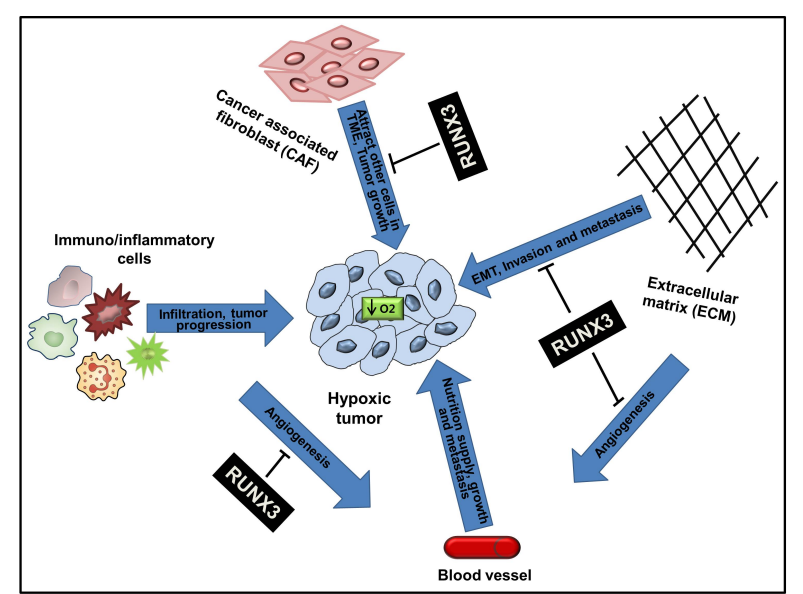

Fig. 1. Role of RUNX3 in the tumor microenvironment and its impact on EMT, tumor growth, invasion and metastasis. A growing tumor lacks oxygen $\left(\mathrm{O}_{2}\right)$ and becomes hypoxic. The hypoxic tumor modulates the tumor microenvironment for its further progression to malignancy. Extracellular matrix (ECM)secreted cytokines facilitate epithelial-mesenchymal transition (EMT) and invasion, increased tumor-infiltrating immune cells suppress immunosurveillance and increase angiogenesis, tumor-modified cancer associated fibroblast (CAF) produce chemokines that attract other tumor-growth-favoring cells into tumor. RUNX3 can repress these phenomena by targeting tumor infiltrating cells, ECM and their secretions.

metastasis (38). Accumulation of TAMs in hypoxic and necrotic zones of the tumor is facilitated by secreted cytokines, including vascular endothelial growth factor (VEGF) in hypoxic regions (39). In addition to that fact, infiltrating immune cells, like T cells, myeloid-derived suppressor cells (MDSCs), and dendritic cells (DCs) within a tumor also pose critical roles in the progression of cancer (2) (Fig. 1).

\section{RUNX3 MEDIATED REGULATION OF INFLAMMATORY CELLS IN TUMOR MICROENVIRONMENT}

Effective immune responses and immunosurveillance of emerging cancer cells are two of the important factors to impede growing tumors. Inflammatory cells have roles at the beginning to prevent tumors through activated innate immune responses, and later a chronic inflammatory state is maintained by infiltrating immune cells, thereby ensuring the survival of tumor cells through intensive crosstalk with tumor cells, leading to its progression (40). Long-time immunocytes are recognized for antitumor abilities; however, recent research shows the involvement of an imbalanced $\mathrm{CD}^{+}{ }^{+} \mathrm{T}$ subset in the tumor immune microenvironment. Runx deregulation is associated with both inflammation and carcinogenesis, including inflammation-induced colorectal cancer (41-43). In a chemicalinduced colorectal cancer model, the expression levels of 
Runx3, but not Runx1 and Runx2, showed a much higher increase in dextran sodium sulfate (DSS)-treated/urokinase-type plasminogen activator (uPA)-deficient mice compared to the wild-type/DSS group. DSS treatment produced significant upregulation of immune cells expressing three Runx proteins identified by the situ investigation. It suggests that immune cells are the main source of Runx proteins and uPA deficiency may be associated with upregulation of the Runx factor in the natural history of inflammatory colon carcinogenesis (44).

RUNX3 facilitates myeloid differentiation through the retinoic acid receptor signaling pathway (45). Furthermore, hematopoietic development in zebrafish is also validated to be regulated by RUNX3 (46). These observations suggest its potential involvement in the regulation of inflammatory cells in immune-tumor microenvironment (2), as well as hematological malignancies. In a large number of tumor types, $\mathrm{T}$ regulatory cells (Tregs) are often found in infiltrating tumors. The rapidly elevated Tregs in the tumor microenvironment is associated with a poor clinical outcome due to their effective immunosuppressive nature i.e., limitation of antitumor immunity, thus promoting cancer progression and angiogenesis (47). Besides, cancer progression, Treg cells are also reported to play a pivotal role in the development and maintenance of terminal immunodeficiency, resulting in serious autoimmune disorders and compartmental defects $(48,49)$. The immune suppressive role of RUNX3 has been reported in Tregs in breast tumors (49). A recent report found that RUNX3, a CD8 ${ }^{+}$ lineage-specific transcription factor, binds to induce transcription of the Forkhead box p3 (FOXP3)-promoter and the $\mathrm{CD}^{+} \mathrm{CD}^{+} 5^{+} \mathrm{FOXP3}^{+}$Treg population is increased in the tumor microenvironment with the progression of breast tumors. Infiltration of $\mathrm{CD}^{+}{ }^{+}$Treg cells in tumor microenvironment produce high-level of immunosuppressive cytokines that ensure repressed effector $\mathrm{T}$ cell proliferation and enhanced tumor immune evasion $(49,50)$. RUNX3 has crucial role in the cytotoxic $\mathrm{T}$ cell maturation. Thymocyte progenitors undergo differentiation through a series of stages and RUNX3 contribute to transcription of $\mathrm{CD} 8$ in $\mathrm{CD} 8{ }^{+} \mathrm{CD} 4{ }^{-} \mathrm{T}$ cells by binding to the CD8 enhancer, thus ensuring maturation into CD8 T cell lineages (51). Natural killer cells (NKC) and cytotoxic lymphocytes infiltrating into solid tumors have highly expressed levels of RUNX3 and play crucial roles in their proliferation and activation. They regulate markers of the effector cytotoxic $\mathrm{T}$ lymphocyte program, including IFN- $\gamma$, perforin, and granzyme B suggesting its critical role in the differentiation of NKC and cytotoxic T-lymphocytes (52). In the report by $\mathrm{Li}$ et al. (53), decreased expression of the Th1associated factor was shown with repressed RUNX3 in gastric carcinoma, as a cause for the cancer progression. IFN- $\gamma$, the specific factor secreted by Th1 cells, regulating the function of target T cells and facilitating the killing of the tumor by NKC, was found repressed with a decreased level of RUNX3 expression (Fig. 1).

Among the growth factors (including cytokines) secreted by a tumor and surrounding interstitial cells, transforming growth factor-beta (TGF- $\beta$ ) might be the most secreted cytokine (54). Secreted TGF- $\beta$ may induce a paradoxical cancer effect by activating an antiproliferative signal or by giving a prooncogenic character to cells, including epithelial-mesenchymal transition (EMT) (54). A recent report suggests that TGF- $\beta$ promotes genomic instability in the form of DNA double-strand breaks (DSB) in cancer cells without RUNX3. The down-regulation of the redox modulator, heme oxygenase- 1 (HO- 1$)$, due to a low concentration of RUNX3, increased oxidative DNA damage and ultimately destroyed genome integrity and triggered cellular senescence accompanied by tumor-promoting inflammatory cytokine expression and acquisition of senescencerelated secretory behavior. Tumor-bearing TGF- $\beta$ gene expression signatures and RUNX3 loss showed higher levels of genomic instabilities (55), suggesting a novel connection between microenvironment-derived extrinsic TGF- $\beta$ signaling and intrinsic RUNX3 inactivation in genomic instability. In addition, TGF- $\beta$ induced EMT is highly noticed in Runx3 null gastric epithelial lines, emphasizing the role of RUNX3 in repression of TGF- $\beta$ induced EMT (56).

ECM provides a scaffold for all cells in TME and has a further diverse role in evolution and spreading of cancer. Proteases like matrix metalloproteinases (MMPs) not only degrade ECM but also help in the remodeling of ECM leading to the secretion of chemokines and other angiogenic and growth factors. RUNX3 has substantiated repressive role over MMPs in various cancers $(12,13,57,58)$, and the loss of RUNX3 was found to be correlated with increased secretion of multifunctional glyco-phosphoprotein osteopontin (OPN), promoting gastric cancer metastasis (59), suggesting its critical role over regulation of TME (Fig. 1).

Nonmalignant cells of TME, like stromal cells and fibroblasts, can secrete growth factors, such as hepatocyte growth factor (HGF), fibroblast growth factor (FGF) and C-X-C motif chemokine ligand 12 (CXCL12) chemokine. These secreted molecules, not only involve in growth and survival of malignant cells, but also involve in recruitment of other cells into the TME, due to their chemoattractant efficacy (33). In a report, CXCL12 is shown to regulate the differentiation of the macrophage by an autocrine/paracrine mechanism which is characterized by expression of the angiogenic factors, VEGF and chemokine (C-C motif) ligand 1 (CCL1). Interestingly, it is further shown to downregulate RUNX3 expression, which maintains CD4 and CD14 expression in mononuclear phagocytes as a part of the transcriptional program induced by CXCL12 (60). Besides, immunosuppressive and proangiogenic functions of VEGF, MDSCs induced by VEGF signaling can play important roles in tumor immune evasion in ovarian cancer (61). In a line VEGF inhibits DC differentiation into mature DC cells (62) and promotes the accumulation of MDSCs, Tregs, and TAMs (61) and RUNX3 can directly inhibit VEGF secretion through transcriptional repression (63) (Fig. 1). These reports suggest RUNX3 as a potential candidate in the 
regulation of the tumor microenvironment, with the ability to suppress activities of tumor promoting cytokines.

\section{HYPOXIA, EPIGENETICS, AND ANGIOGENESIS}

The hypoxic environment is created due to an increase in the tumor size followed by the subsequent inadequate supply of oxygen in a growing tumor and further encompasses metabolic and biological processes, making them more aggressive and irresponsive to therapeutic interventions (64). Growing body of evidences have shown the role of hypoxia in maintenance of cancer stem cells (CSCs), through repressed tumor cell differentiation and increased therapeutic resistance thus assuring tumor progression (65). In other report, hypoxia mediated repression of mesenchymal stem and progenitor cell differentiation is substantiated as a critical factor in the evolution of a tumor stromal microenvironment, the putative cancer stem cell niches (66). In keeping with this fact, hypoxia upregulates hypoxia-inducible factor 1 alpha (HIF-1 $\alpha$ ) that induces angiogenesis, and it is associated with activation of genes involved in metastasis (13), such as increased cell migration and ECM remodeling (3).

In various cancer tissues, RUNX3 is silenced due to promoter DNA hyper methylation. However, the effect of the hypoxic tumor microenvironment in the regulation of RUNX3 is obscure. Our group has described a mechanism for the first time to silencing of RUNX3 due to histone modification under hypoxic microenvironment. Histone methylation and deacetylation occur under the influence of histone methyltransferase (HMT) G9a and histone deacetylase (HDAC) 1 respectively (67). HMT G9a is upregulated by hypoxia (68) and is responsible for poor prognosis and metastasis of several human cancers (69-72). Hypoxia can also activate HDAC1 (73). Acetylation of RUNX3 by p300 (74) and bromodomain (BRD) (75) is a key to the protein stability and cell-cycle arrest. Thus, RUNX3 inactivation could also be due to the histone deacetylation. In support of this, HDAC inhibitors restored RUNX3 expression and subsequent tumor-suppressive character in cancer cells $(67,76,77)$. Therefore, it can be of critical importance to rescue epigenetic loss of RUNX3 expression to help to prevent cancer cell from facilitated by hypoxic tumor microenvironment.

Small noncoding RNAs, microRNAs (miRNAs), are the important molecule that plays critical roles in posttranscriptional regulation of genes affecting diverse biological processes in a large number of cell types (78), along with their critical roles as tumor suppressors or promoters. Specific groups of miRNA are regulated by hypoxia (79). Emerging evidences have shown that miRNAs regulated tumor microenvironment influencing tumor immune invasion, tumor angiogenesis and tumor-stromal interaction $(80,81)$. However, upregulated RUNX3 expression is identified in epithelial ovarian cancer due to repressed miR130b expression and subsequent promotion of carcinogenic feature (82), in GC, our group has shown silencing of RUNX3 by miR-130a/miR-495 for the early tumorigenic progression due to increased VEGF secretion in hypoxic status (83). These reports suggest probable manipulation of the tumor microenvironment by targeting RUNX3.

Dynamic shuttling of RUNX3 between nuclear and cytoplasmic compartments is tightly linked with nuclear components, signifying probable importance of targeting RUNX3 to the nuclear matrix for active regulation and biological consequences (84). In support of this, threonine 209 phosphorylation on RUNX3 by p21 activated kinase 1 (Pak1) translocate it from nucleus to the cytoplasm and subsequently converses its biological functions (85). In another report, provirus integration site for Moloney murine leukemia virus 1 (pim-1) was shown to phosphorylate RUNX3 and alter its expression by mislocalization in salivary gland adenoid cystic carcinoma (ACC) (86). Besides mislocalization and inactivation of RUNX3, ubiquitination and subsequent proteasomal degradation are also substantiated for its mode of inactivation in GC infected by virulent cytotoxin-associated gene A (CagA)-positive Helicobacter pylori. CagA can bind to RUNX3, inducing ubiquitination and subsequent degradation of RUNX3 by proteasomal machinery (87). In response to these reports, we can suggest that blocking mislocalization and ubiquitination of RUNX3 could be of importance in tumors to prevent from advancing to malignancy.

In recent years, a number of reports have shown evidence of hypoxic stress in the tumor microenvironment, playing a prominent role in tumor immune responses. Upregulated HIF- $1 \alpha$ in hypoxic environment facilitates the immunesuppressive ability of MDSC and TAM and augment differentiation of MDSC to TAM (88). Under hypoxic conditions, RUNX3 decreases the half-life of HIF-1 $\alpha$, its stability, transactivation activity and VEGF secretion due to repressed nuclear localization. Furthermore, overexpression of RUNX3 significantly inhibits hypoxia-induced angiogenesis (89). The critical role played by RUNX3 in inflammatory cells have also been emphasized by some authors, rather than its tumor suppressive role (43). On this note, we can speculate that forcefully targeting RUNX3 to over express in a hypoxic tumor could be a promising intervention to regulate and activate immune responses, and modulate ECM and CAF in TME to inhibit tumor growth, migration, invasion and angiogenesis (Fig. 1).

However, RUNX3 is established as a potent tumor suppressor in GC and some other forms of the tumor; the oncogenic behavior of RUNX3 has also been specified. Patients with oral squamous cell carcinoma (OSCC) are identified for high occurrence and low survival rates. Furthermore, mice subcutaneously inoculated with RUNX3 knockdown OSCC verified for the reduced bone invasion and production of osteolytic factors (19). In another report RUNX3 restoration in OSCC cells illustrated to suppress cell migration and invasion by downregulating MMP-9 expression and 
secretion, thus potentiating antiangiogenic behavior by inhibiting VEGF activity (90). In a model of skin tumorigenesis, leukocytic loss of RUNX3 suppressed protumorigenic cytokines, interleukin-17a (IL-17a) and OPN substantially decreasing carcinogen-induced skin tumorigenesis (91). In head and neck squamous cell carcinoma (HNSCC), overexpressed RUNX3 is detected as a critical reason for a malignant phenotype which is caused, in part, due to demethylation during cancer progression (17). These reports reflect on the organ-specific and contextual based behavior of RUNX3.

\section{IMPLICATIONS FOR CANCER THERAPY}

RUNX3 expressed in T cells have an important role in the functioning of the immune system. It has been reported that mice deficient in RUNX3 spontaneously develop immunological abnormalities like airway hypersensitivities, colitis and also gastric hyperplasia $(92,93)$. Likewise, T-cell-targeted knockdown in mice spontaneously develop asthma-like symptoms with infiltration of lymphocytes in the lungs (94). Reduced RUNX3 in gastric carcinoma is positively correlated with repressed Th1-associated factor and decreased Th1 cell-mediated immunity is associated with immune escape of cancer cells $(52,53)$. In addition, miRNA-145 could regulate the balance of Th1/Th2 through targeting RUNX3 in asthma patients, and miRNA-145 and RUNX3 can be used as biomarkers or targets in the diagnosis or therapy of asthma (95). On this note, it can be of great significance to target RUNX3 for the prevention of immunological disease, including the immune escape of cancer cells.

Leukocyte infiltration into tumors of a certain size is interconnected with tumor angiogenesis. Pro-angiogenic factor VEGF and related molecules are effective monocyte attractants leading to the monocytes recruitment into tumors advancing to metastasis (reviewed in (96)). Thus, the inhibition of leukocyte recruitment could be of therapeutic importance to suppress the tumor angiogenesis. As increased expression of RUNX3 is determined to be needed for VEGF suppression in OSCC (90), it can be speculated that targeting RUNX3 could be a potent molecular tool to prevent tumor growth and angiogenesis through repressing leukocyte infiltration into tumors.

\section{CONCLUSION}

Specifically, in GC and many other forms of cancer, RUNX3 suppression through different microenvironmental states, including hypoxia, is highlighted for cancer initiation, invasion and metastasis. While in some cancers, RUNX3 is illustrated oncogene-enhancing cancer cell proliferation and invasion. The involvement of RUNX3 in immune cell maintenance and regulation has forced researchers to address RUNX3 as a tumor immune microenvironmental regulator, rather than focus only on an appreciation of its role as a tumor suppressor. As TME has inevitable importance in the progression of cancer, the role of RUNX3 must be further explored in order to bolster early findings of its potential as a target for the effective treatment of cancer.

\section{ACKNOWLEDGEMENTS}

This work was supported by NRF-2017R1D1A3B03034382 (to S.M.), and NRF-2017R1A2B3002227 (to Y.M.L).

\section{CONFLICTS OF INTEREST}

The authors have no conflicting interests.

\section{REFERENCES}

1. Klemm F and Joyce JA (2015) Microenvironmental regulation of therapeutic response in cancer. Cell Biol 25, 198-213

2. Balkwill FR, Capasso M and Hagemann T (2012) The tumor microenvironment at a glance. J Cell Sci 125, 5591-5596

3. Hockel $M$ and Vaupel P (2001) Tumor hypoxia: definitions and current clinical, biologic, and molecular aspects. J Natl Cancer Inst 93, 266-276

4. Ito Y (1999) Molecular basis of tissue-specific gene expression mediated by the runt domain transcription factor PEBP2/CBF. Genes Cells 4, 685-696

5. Ito Y (2008) RUNX genes in development and cancer: regulation of viral gene expression and the discovery of RUNX family genes. Adv Cancer Res 99, 33-76

6. Kundu M, Compton S, Garrett-Beal L et al (2005) Runx1 deficiency predisposes mice to T-lymphoblastic lymphoma. Blood 106, 3621-3624

7. Ito $Y$ (2004) Oncogenic potential of the RUNX gene family: 'overview'. Oncogene 23, 4198-4208

8. Leong DT, Lim J, Goh X et al (2010) Cancer-related ectopic expression of the bone-related transcription factor RUNX2 in non-osseous metastatic tumor cells is linked to cell proliferation and motility. Cancer Res 12, R89

9. Woolf E, Xiao C, Fainaru O et al (2003) Runx3 and Runx1 are required for CD8 $\mathrm{T}$ cell development during thymopoiesis. Proc Natl Acad Sci U S A 100, 7731-7736

10. Levanon D, Bettoun D, Harris-Cerruti C et al (2002) The Runx3 transcription factor regulates development and survival of TrkC dorsal root ganglia neurons. EMBO J 21, 3454-3463

11. Bae SC and Choi JK (2004) Tumor suppressor activity of RUNX3. Oncogene 23, 4336-4340

12. Chen F, Bai J, Li W et al (2013) RUNX3 suppresses migration, invasion and angiogenesis of human renal cell carcinoma. PLoS One 8, e56241

13. Chen F, Wang M, Bai J et al (2014) Role of RUNX3 in suppressing metastasis and angiogenesis of human prostate cancer. PLoS One 9, e86917

14. Chuang LS and Ito $Y$ (2010) RUNX3 is multifunctional in carcinogenesis of multiple solid tumors. Oncogene 29, 2605-2615

15. Kim HJ, Park J, Lee SK, Kim KR, Park KK and Chung WY 
(2015) Loss of RUNX3 expression promotes cancerassociated bone destruction by regulating CCL5, CCL19 and CXCL11 in non-small cell lung cancer. J Pathol 237, 520-531

16. Li QL, Ito K, Sakakura C et al (2002) Causal relationship between the loss of RUNX3 expression and gastric cancer. Cell 109, 113-124

17. Kudo $Y$, Tsunematsu $T$ and Takata $T$ (2011) Oncogenic role of RUNX3 in head and neck cancer. J Cell Biochem $112,387-393$

18. Lee CW, Chuang LS, Kimura $S$ et al (2011) RUNX3 functions as an oncogene in ovarian cancer. Gynecol Oncol 122, 410-417

19. Park J, Kim HJ, Kim KR et al (2017) Loss of RUNX3 expression inhibits bone invasion of oral squamous cell carcinoma. Oncotarget 8, 9079-9092

20. Inoue K, Ozaki S, Shiga T et al (2002) Runx3 controls the axonal projection of proprioceptive dorsal root ganglion neurons. Nat Neurosci 5, 946-954

21. Lau QC, Raja E, Salto-Tellez M et al (2006) RUNX3 is frequently inactivated by dual mechanisms of protein mislocalization and promoter hypermethylation in breast cancer. Cancer Res 66, 6512-6520

22. Ito K, Liu Q, Salto-Tellez M et al (2005) RUNX3, a novel tumor suppressor, is frequently inactivated in gastric cancer by protein mislocalization. Cancer Res 65, 7743-7750

23. Li QL, Kim HR, Kim WJ et al (2004) Transcriptional silencing of the RUNX3 gene by CpG hypermethylation is associated with lung cancer. Biochem Biophys Res Commun 314, 223-228

24. Kim WJ, Kim EJ, Jeong P et al (2005) RUNX3 inactivation by point mutations and aberrant DNA methylation in bladder tumors. Cancer Res 65, 9347-9354

25. Zhang X, Wang L, Zeng X, Fujita T and Liu W (2017) Runx3 inhibits melanoma cell migration through regulation of cell shape change. Cell Biol International 41, 1048-1055

26. Cameron ER and Neil JC (2004) The Runx genes: lineage-specific oncogenes and tumor suppressors. Oncogene 23, 4308-4314

27. Salto-Tellez M, Peh BK, Ito K et al (2006) RUNX3 protein is overexpressed in human basal cell carcinomas. Oncogene 25, 7646-7649

28. Tsunematsu T, Kudo Y, lizuka $S$ et al (2009) RUNX3 has an oncogenic role in head and neck cancer. PLoS One 4, e5892

29. Bledsoe KL, McGee-Lawrence ME, Camilleri ET et al (2014) RUNX3 facilitates growth of Ewing sarcoma cells. J Cell Physiol 229, 2049-2056

30. Hanahan D and Weinberg RA (2000) The hallmarks of cancer. Cell 100, 57-70

31. Hanahan D and Weinberg RA (2011) Hallmarks of cancer: the next generation. Cell 144, 646-674

32. Quail DF and Joyce JA (2013) Microenvironmental regulation of tumor progression and metastasis. Nat Med $19,1423-1437$

33. Hanahan D and Coussens LM (2012) Accessories to the crime: functions of cells recruited to the tumor microenvironment. Cancer Cell 21, 309-322
34. Weber CE and Kuo PC (2012) The tumor microenvironment. Surg Oncol 21, 172-177

35. Blagosklonny MV (2004) Antiangiogenic therapy and tumor progression. Cancer Cell 5, 13-17

36. Semenza GL (2000) Hypoxia, clonal selection, and the role of HIF-1 in tumor progression. Crit Rev Biochem Mol Biol 35, 71-103

37. Qian BZ and Pollard JW (2010) Macrophage diversity enhances tumor progression and metastasis. Cell 141, 39-51

38. Condeelis J and Pollard JW (2006) Macrophages: obligate partners for tumor cell migration, invasion, and metastasis. Cell 124, 263-266

39. Murdoch C, Giannoudis A and Lewis CE (2004) Mechanisms regulating the recruitment of macrophages into hypoxic areas of tumors and other ischemic tissues. Blood 104, 2224-2234

40. Grivennikov SI, Greten FR and Karin M (2010) Immunity, inflammation, and cancer. Cell 140, 883-899

41. Chuang LS, Ito $K$ and Ito $Y$ (2013) RUNX family: Regulation and diversification of roles through interacting proteins. Int J Cancer 132, 1260-1271

42. Ito Y, Bae SC and Chuang LS (2015) The RUNX family: developmental regulators in cancer. Nat Rev Cancer 15, 81-95

43. Lotem J, Levanon D, Negreanu V et al (2015) Runx3 at the interface of immunity, inflammation and cancer. Biochim Biophys Acta 1855, 131-143

44. Afaloniati $\mathrm{H}$, Karagiannis GS, Hardas A, Poutahidis $\mathrm{T}$ and Angelopoulou K (2017) Inflammation-driven colon neoplasmatogenesis in uPA-deficient mice is associated with an increased expression of Runx transcriptional regulators. Exp Cell Res 361, 257-264

45. Le XF, Groner Y, Kornblau SM et al (1999) Regulation of AML2/CBFA3 in hematopoietic cells through the retinoic acid receptor alpha-dependent signaling pathway. J Biol Chem 274, 21651-21658

46. Kalev-Zylinska ML, Horsfield JA, Flores MV et al (2003) Runx3 is required for hematopoietic development in zebrafish. Dev Dyn 228, 323-336

47. Facciabene A, Motz GT and Coukos G (2012) T-regulatory cells: key players in tumor immune escape and angiogenesis. Cancer Res 72, 2162-2171

48. Hossain DM, Panda AK, Chakrabarty S et al (2015) MEK inhibition prevents tumour-shed transforming growth factor-beta-induced T-regulatory cell augmentation in tumour milieu. Immunology 144, 561-573

49. Dinesh RK, Skaggs BJ, La Cava A, Hahn BH and Singh RP (2010) CD8 + Tregs in lupus, autoimmunity, and beyond. Autoimm Rev 9, 560-568

50. Chakraborty S, Panda AK, Bose S et al (2017) Transcriptional regulation of FOXP3 requires integrated activation of both promoter and CNS regions in tumor-induced CD8(+) Treg cells. Sci Rep 7, 1628

51. Egawa T, Tillman RE, Naoe $Y$, Taniuchi I and Littman DR (2007) The role of the Runx transcription factors in thymocyte differentiation and in homeostasis of naive T cells. J Exp Med 204, 1945-1957

52. Cruz-Guilloty F, Pipkin ME, Djuretic IM et al (2009) Runx3 and T-box proteins cooperate to establish the 
transcriptional program of effector CTLs. J Exp Med 206, 51-59

53. Li Y, Ji X, Su Z et al (2014) Downregulation of Runx3 is closely related to the decreased Th1-associated factors in patients with gastric carcinoma. Tumour Biol 35, 12235-12244

54. Massague J (2008) TGFbeta in Cancer. Cell 134, 215-230

55. Krishnan V, Chong YL, Tan TZ et al (2018) TGF-beta promotes genomic instability after loss of RUNX3. Cancer Res 78, 88-102

56. Voon DC, Hor YT and Ito Y (2015) The RUNX complex: reaching beyond haematopoiesis into immunity. Immunology 146, 523-536

57. Mei PJ, Bai J, Liu H et al (2011) RUNX3 expression is lost in glioma and its restoration causes drastic suppression of tumor invasion and migration. J Cancer Res Clin Oncol $137,1823-1830$

58. Kim BR, Kang MH, Kim JL et al (2016) RUNX3 inhibits the metastasis and angiogenesis of colorectal cancer. Oncol Rep 36, 2601-2608

59. Cheng HC, Liu YP, Shan YS et al (2013) Loss of RUNX3 increases osteopontin expression and promotes cell migration in gastric cancer. Carcinogenesis 34, 2452-2459

60. Sanchez-Martin L, Estecha A, Samaniego R, SanchezRamon S, Vega MA and Sanchez-Mateos P (2011) The chemokine CXCL12 regulates monocyte-macrophage differentiation and RUNX3 expression. Blood 117, 88-97

61. Horikawa N, Abiko K, Matsumura N et al (2017) Expression of Vascular Endothelial Growth Factor in Ovarian Cancer Inhibits Tumor Immunity through the Accumulation of Myeloid-Derived Suppressor Cells. Clin Cancer Res 23, 587-599

62. Oussa NA, Dahmani A, Gomis M, Richaud M, Andreev E and Navab-Daneshmand AR (2016) VEGF Requires the Receptor NRP-1 To Inhibit Lipopolysaccharide-Dependent Dendritic Cell Maturation. J Immunol 197, 3927-3935

63. Peng Z, Wei D, Wang L et al (2006) RUNX3 inhibits the expression of vascular endothelial growth factor and reduces the angiogenesis, growth, and metastasis of human gastric cancer. Clin Cancer Res 12, 6386-6394

64. Gordan JD, Thompson CB and Simon MC (2007) HIF and c-Myc: sibling rivals for control of cancer cell metabolism and proliferation. Cancer Cell 12, 108-113

65. Simon MC and Keith B (2008) The role of oxygen availability in embryonic development and stem cell function. Nat Rev Mol Cell Biol 9, 285-296

66. Lin Q and Yun Z (2010) Impact of the hypoxic tumor microenvironment on the regulation of cancer stem cell characteristics. Cancer Biol Ther 9, 949-956

67. Lee SH, Kim J, Kim WH and Lee YM (2009) Hypoxic silencing of tumor suppressor RUNX3 by histone modification in gastric cancer cells. Oncogene 28, 184-194

68. Chen H, Yan Y, Davidson TL, Shinkai $Y$ and Costa $M$ (2006) Hypoxic stress induces dimethylated histone H3 lysine 9 through histone methyltransferase G9a in mammalian cells. Cancer Res 66, 9009-9016

69. Chen MW, Hua KT, Kao HJ et al (2010) H3K9 histone methyltransferase G9a promotes lung cancer invasion and metastasis by silencing the cell adhesion molecule Ep-CAM. Cancer Res 70, 7830-7840

70. Liu S, Ye D, Guo W et al (2015) G9a is essential for EMT-mediated metastasis and maintenance of cancer stem cell-like characters in head and neck squamous cell carcinoma. Oncotarget 6, 6887-6901

71. Dong C, Wu Y, Yao J et al (2012) G9a interacts with Snail and is critical for Snail-mediated E-cadherin repression in human breast cancer. J Clin Invest 122, 1469-1486

72. Yuan Y, Tang AJ, Castoreno AB et al (2013) Gossypol and an HMT G9a inhibitor act in synergy to induce cell death in pancreatic cancer cells. Cell Death Dis 4, e690

73. Kim MS, Kwon HJ, Lee YM et al (2001) Histone deacetylases induce angiogenesis by negative regulation of tumor suppressor genes. Nat Med 7, 437-443

74. Iwatani K, Fujimoto T and Ito T (2010) Cyclin D1 blocks the anti-proliferative function of RUNX3 by interfering with RUNX3-p300 interaction. Biochem Biophys Res Commun 400, 426-431

75. Lee YS, Lee JW, Jang JW et al (2013) Runx3 inactivation is a crucial early event in the development of lung adenocarcinoma. Cancer Cell 24, 603-616

76. Huang C, Ida H, Ito $K$, Zhang $H$ and Ito $Y$ (2007) Contribution of reactivated RUNX3 to inhibition of gastric cancer cell growth following suberoylanilide hydroxamic acid (vorinostat) treatment. Biochem Pharmacol 73, 990-1000

77. Shio S, Kodama Y, Ida $\mathrm{H}$ et al (2011) Loss of RUNX3 expression by histone deacetylation is associated with biliary tract carcinogenesis. Cancer Sci 102, 776-783

78. He L and Hannon GJ (2004) MicroRNAs: small RNAs with a big role in gene regulation. Nat Rev Genetics 5, 522-531

79. Kulshreshtha R, Davuluri RV, Calin GA and Ivan M (2008) A microRNA component of the hypoxic response. Cell Death Differ 15, 667-671

80. Nicoloso MS, Spizzo R, Shimizu M, Rossi S and Calin GA (2009) MicroRNAs-the micro steering wheel of tumour metastases. Nat Rev Cancer 9, 293-302

81. Wentz-Hunter KK and Potashkin JA (2011) The Role of miRNAs as Key Regulators in the Neoplastic Microenvironment. Mol Biol Int 2011, 839872

82. Paudel D, Zhou W, Ouyang Y et al (2016) MicroRNA$130 \mathrm{~b}$ functions as a tumor suppressor by regulating RUNX3 in epithelial ovarian cancer. Gene 586, 48-55

83. Lee SH, Jung YD, Choi YS and Lee YM (2015) Targeting of RUNX3 by miR-130a and miR-495 cooperatively increases cell proliferation and tumor angiogenesis in gastric cancer cells. Oncotarget 6, 33269-33278

84. Pande S, Ali SA, Dowdy C et al (2009) Subnuclear targeting of the Runx3 tumor suppressor and its epigenetic association with mitotic chromosomes. J Cell Phys 218, 473-479

85. Kumar A, Singhal M, Chopra C et al (2016) Threonine 209 phosphorylation on RUNX3 by Pak1 is a molecular switch for its dualistic functions. Oncogene 35, 4857-4865

86. Zhu X, Xu JJ, Hu SS et al (2014) Pim-1 acts as an oncogene in human salivary gland adenoid cystic carcinoma. J Exp Clin Cancer Res 33, 114 
87. Tsang YH, Lamb A, Romero-Gallo J et al (2010) Helicobacter pylori CagA targets gastric tumor suppressor RUNX3 for proteasome- mediated degradation. Oncogene 29, 5643-5650

88. Kumar V and Gabrilovich DI (2014) Hypoxia-inducible factors in regulation of immune responses in tumour microenvironment. Immunology 143, 512-519

89. Lee SH, Bae SC, Kim KW and Lee YM (2014) RUNX3 inhibits hypoxia-inducible factor-1alpha protein stability by interacting with prolyl hydroxylases in gastric cancer cells. Oncogene 33, 1458-1467

90. Zhou WN, Du YF, Bai J et al (2017) RUNX3 plays a tumor suppressor role by inhibiting cell migration, invasion and angiogenesis in oral squamous cell carcinoma. Oncol Rep $38,2378-2386$

91. Bauer O, Hantisteanu S, Lotem J and Groner Y (2014) Carcinogen-induced skin tumor development requires leukocytic expression of the transcription factor Runx3.
Cancer Pre Res (Phila) 7, 913-926

92. Brenner O, Levanon D, Negreanu $V$ et al (2004) Loss of Runx3 function in leukocytes is associated with spontaneously developed colitis and gastric mucosal hyperplasia. Proc Nat Acad Sci U S A 101, 16016-16021

93. Fainaru O, Woolf $E$, Lotem J et al (2004) Runx3 regulates mouse TGF-beta-mediated dendritic cell function and its absence results in airway inflammation. EMBO J 23, 969-979

94. Naoe Y, Setoguchi R, Akiyama K et al (2007) Repression of interleukin-4 in T helper type 1 cells by Runx/Cbf beta binding to the II4 silencer. J Exp Med 204, 1749-1755

95. Fan L, Wang X, Fan L et al (2016) MicroRNA-145 influences the balance of Th1/Th2 via regulating RUNX3 in asthma patients. Exp Lung Res 42, 417-424

96. Mantovani A, Allavena P, Sica A and Balkwill F (2008) Cancer-related inflammation. Nature 454, 436-444 